University of Warwick institutional repository: http://go.warwick.ac.uk/wrap This paper is made available online in accordance with publisher policies. Please scroll down to view the document itself. Please refer to the repository record for this item and our policy information available from the repository home page for further information.

To see the final version of this paper please visit the publisher's website. access to the published version may require a subscription.

Author(s): Traolach S. Brugha, Scott Weich, Nicola Singleton , Glyn Lewis, Paul E. Bebbington, Rachel Jenkins and Howard Meltzer Article Title: Primary group size, social support, gender and futuremental health status in a prospective study of people living in private households throughout Great Britain Year of publication: 2005 Link to published version:http://dx.doi.org/ 10.1017/S0033291704003903

Publisher statement: None 


\title{
Primary group size, social support, gender and future mental health status in a prospective study of people living in private households throughout Great Britain
}

\author{
TRAOLACH S. BRUGHA ${ }^{1 *}$, SCOTT WEICH ${ }^{2}$, NICOLA SINGLETON ${ }^{3}$, \\ GLYN LEWIS ${ }^{4}$, PAUL E. BEBBINGTON ${ }^{5}$, RACHEL JENKINS ${ }^{6}$ \\ AND HOWARD MELTZER ${ }^{7}$
}

${ }^{1}$ University of Leicester, Department of Health Sciences, Brandon Mental Health Unit, Leicester General Hospital, Leicester, UK $;{ }^{2}$ Division of Health in the Community, Warwick Medical School (LWMS), University of Warwick, Coventry, UK $;{ }^{3}$ Home Office, 50 Queen Anne's Gate, London, UK $;{ }^{4}$ University of Wales College of Medicine, Department of Psychological Medicine, Heath Park, Cardiff, UK; ${ }^{5}$ Royal Free and University College London Medical School, Department of Psychiatry and Behavioural Sciences, Highgate Hill, London, UK $;{ }^{6}$ WHO Collaborating Centre, Institute of Psychiatry, De Crespigny Park, London, UK;

${ }^{7}$ Office for National Statistics, 1 Drummond Gate, London, UK

\begin{abstract}
Background. Structural characteristics of social networks such as primary group size have received less attention than measures of perceived social support. Previous research suggests that associations between social network size and later common mental disorder status may differ according to sex and initial mental state.
\end{abstract}

Method. Adults participating in the 2000 British National Household Survey of psychiatric morbidity were randomly selected for follow-up 18 months later. The revised Clinical Interview Schedule (CIS-R) and the Interview Measure of Social Relations (IMSR) were administered at baseline and follow-up. Primary group size was defined as the total number of close relatives and friends. A four-level scale of common mental disorder was modelled with ordinal logistic regression, based on weighted data $(n=2413)$.

Findings. After adjusting for confounders, a primary group size of three or less at time 1 predicted worse mental health at time 2. This effect was greatest in men who were initially non-cases at baseline (averaged odds 4.5) and in women who were initially cases at baseline (average odds $2 \cdot 9$ ). Primary group size at time 2 was significantly predicted by level of common mental disorder at time 1 in women but not in men. Thus, confounding by baseline disorder does not explain risk of developing poor mental health in socially isolated men.

Conclusion. This study replicates the strong effects of primary group size on future mental health that emerge when men and women are studied separately and when subjects are categorized according to baseline mental health status.

\section{INTRODUCTION}

\section{Background}

Structural characteristics of social networks such as their size have received less attention

* Address for correspondence: Dr Traolach S. Brugha, University of Leicester, Department of Health Sciences, Brandon Mental Health Unit, Leicester General Hospital, Leicester LE5 4PW, UK. (Email: tsb@1e.ac.uk) than measures of perceived social support Brugha et al. (2003). In 1978 Scott Henderson showed that the size of the primary group, those felt to be close in a person's social support network, was significantly smaller in psychiatric outpatients than in community controls (Henderson et al. 1978). These findings were replicated by Brugha et al. (1982), and also by Meltzer and 
colleagues (1995a), who reported data from the first British National Survey of Psychiatric Morbidity.

There is evidence that the association of the size of the social network with common mental disorders (CMD) may vary with sex, particularly in studies of course and outcome. Thus, depressed female psychiatric patients with small primary groups had a significantly worse clinical outcome approximately 4 months later, but this was not true of males (Brugha et al. 1990). A recent prospective study in a community sample of 32 women with major depression (Wildes et al. 2002) found that the total number of close confiding relationships was a significantly stronger predictor of depression approximately 1 year later than were life events. However, the possible importance of a second distinction, that between symptom development and prognosis, came with the finding that primary group size assessed antenatally in the community showed no relation to the emergence of CMD or depression in the 3 months after childbirth (Brugha et al. 1998), although dissatisfaction with support from others did. In a crosssectional community study in Finland (Hintikka et al. 2000), there were marked differences between the sexes, as only in men was the number of close friends associated with the risk of mental distress. Studies of loneliness also suggest that social network group size in community populations is more important for men than for women (Stokes \& Levin, 1986). Other researchers have used different definitions and measures of social network size to examine interactions with sex, although without distinguishing symptom development and prognosis (Brugha \& Britto, 1992; Kawachi \& Berkman, 2001).

Social network size may, therefore, have a greater bearing on the risk of developing mental ill health in men than in women, but it is possible that this gender effect is reversed when we look at the prognosis of those who are already assessed as cases. Four studies suggest that in women who are cases a small network of close others predicts a worse prognosis (Brugha et al. 1990; Wildes et al. 2002) but that it does not place women who are well at risk of developing disorder (Stokes \& Levin, 1986; Brugha et al. 1998). There is less information on men but it appears that the opposite may apply: men who are well but isolated are at risk of developing disorder but the prognosis for men who are cases is not predicted by the size of their close social network.

An 18-month follow-up of a national survey of people living in private households in Britain in 2000 (Singleton et al. 2001) provided an opportunity to study interactions between primary group size, gender and baseline mental health status in a community sample. Because symptoms and social networks were assessed at both interviews, it was also possible to examine reverse causality in the association between primary group size and the prevalence of the CMD (Paykel \& Weissman, 1973). We compared the effect of primary group size with a separate assessment of perceived social support, which has not generally been shown to interact with gender.

\section{Hypotheses}

We tested the following hypotheses:

(1) After controlling for initial symptom level, gender and other potential confounders, a small primary group at time 1 will predict the severity of CMD at time 2 .

(2) Small primary group size will predict the prevalence of CMD at time 2 in men who are non-cases, and women who are cases, of CMD at baseline (time 1).

(3) The association between perceived social support at time 1 to symptoms at time 2 will not differ significantly between men and women.

\section{METHOD \\ Design}

Full details of the design and sampling have appeared elsewhere (Singleton \& Lewis, 2003). The design involved establishing mental health status at two points in time. At time 1, addresses were sampled from sectors within regions throughout England, Scotland and Wales in 2000, stratified for socio-economic status. One adult aged between 16 and 74 years was selected at random per household yielding 8886 cooperating adults, a response rate of $69 \%$ (Singleton et al. 2001). The sample for the 18-month follow-up included three groups of people, selected on the basis of their mental 
health status at the time of the original national survey of people living in private households, was (with proportion selected):

(1) All people who reported symptoms of CMD sufficient to obtain a score of $12+$ on the instrument used to assess these disorders in the survey, the revised version of the Clinical Interview Schedule (CIS-R; Lewis et al. 1992) and, therefore, identified as a case of mental disorder at time 1 ( 1 in 1 were selected).

(2) People without a mental disorder at time 1 but who did obtain a score of $6-11$ on the CIS-R ( 1 in 1 were selected).

(3) Those people without disorder at time 1 and with little evidence of symptoms of CMD (i.e. with CIS-R scores of $0-5$ ) ( 1 in 5 were selected).

In these three groups respectively the number selected was: 2026, 1212, 1068. Ninety-four per cent of follow-up interviews were conducted within 18 months $( \pm 2$ months) of the baseline interview.

\section{Procedures}

Initial contact with respondents was made by letter; if this failed, the interviewer would try by phone to arrange a visit. A proportion of those who had moved were traced to their new address and asked to take part. Information was collected face-to-face using computer-assisted interviewing (CAI). The follow-up interview was based on a modified version of the questionnaire used in the baseline survey (Singleton et al. 2001).

Data collection was carried out by interviewers from the fieldwork team of the Office for National Statistics (ONS). These were nonclinically trained, experienced survey interviewers who had participated in a one-day projectspecific training programme in the use of the survey instruments. Most had also participated in earlier surveys of psychiatric morbidity conducted by the ONS. Fieldwork was closely monitored by supervisors in the field and by headquarters staff.

\section{Assessment}

At baseline and follow-up ONS interviewers administered a sociodemographic questionnaire covering information, for example on accommodation, educational qualifications, age and income. They assessed the CMD using the CIS-R (we used data on disorder severity and case status and not on disorder type).

Primary group size was assessed with a series of questions from the Interview Measure for Social Relationships (IMSR) on the numbers of friends and relatives (aged 16 years and over) respondents felt close to (Brugha et al. 1987). Data were collected about three groups of people:

(1) adults who lived with respondents and whom respondents felt close to;

(2) relatives who did not live with respondents, but whom they felt close to;

(3) friends or acquaintances who did not live with respondents but who were described as close or good friends.

The total number of close friends and relatives form an individual's 'primary support group' (Henderson et al. 1978; Brugha et al. 1982); equal weight is given to particularly close relationships, such as an attachment figure or partner, close relatives, good friends within or outside the household. Frequency of social contact was not assessed. Adults with a total primary group size of three people or fewer have been shown previously to be at greatest risk of psychiatric morbidity (Brugha et al. 1993).

Perceived social support was assessed from respondents' answers to seven questions taken from the 1987 Health and Lifestyle survey (Cox et al. 1987). The seven questions take the form of statements that individuals could say were 'not true', 'partly true', or 'certainly true' of their family and friends. Scores of 1-3 were obtained for each question and overall scores ranged from 7 to 21 . The maximum score of 21 indicated no lack of social support; scores of 18-20 indicated a moderate lack of social support and scores of 17 and below showed that individuals perceived a severe lack of perceived social support. The seven statements are:

There are people I know - amongst my family or friends -

(1) Who do things to make me happy.

(2) Who make me feel loved.

(3) Who can be relied on no matter what happens.

(4) Who would see that I am taken care of if I needed to be.

(5) Who accept me just as I am. 
(6) Who make me feel an important part of their lives.

(7) Who give me support and encouragement.

Descriptive statistics for the time 1 and time 2 samples, including demographic characteristics, CIS-R scores, social network size, and perceived social support detailed in earlier reports (Singleton et al. 2001; Meltzer et al. 2002; Singleton \& Lewis, 2003; see also ONS, 2004).

\section{Weighting and adjustment procedures}

Data from the baseline survey were weighted when analysed to take account of the varying probability of selection for individuals in different areas of the country and in different sized households. In order to take account of the differential non-response that occurred by region, sex and age group the data were also post-stratified to population control totals to provide a weight that rendered survey estimates representative of the GB population as a whole. Further weighting at follow-up was necessary to take account, first, of the different probability of selection of people in different sample groups, and secondly, non-response. The first stage of the weighting adjusted for the different sampling fractions in the sample groups (described above). For the second stage of weighting, an analysis was carried out using SPSS Answer Tree (SPSS Inc., Chicago, IL, USA) to identify groups of respondents with significantly different rates of non-response. Details of the calculation of the weights used are given in the survey report (Singleton \& Lewis, 2003).

\section{Analysis}

Analyses were based on the weighted data, performed using the survey commands in STATA 6.0 (StataCorp, 1999), which gives more precision of parameter estimates [and thus, confidence intervals (CIs)] when using survey data incorporating probability weights. Clinical outcome was expressed as an ordered scale to reflect severity of disorder. The CIS-R was used to establish an overall symptom score that can in theory range between 0 and 57 . The CIS-R total score at time 1 and at time 2 was divided into four groups (and 3 cut-points): $0-5,6-11$, $12-17, \geqslant 18$. The time 2 score was employed as the dependent variable in ordinal logistic regression models, using the STATA command SVYOLOGIT, with primary group size, lack of perceived social support, age group and CIS-R grouped total score assessed at time 1 as predictors. With ordinal logistic regression (proportional odds modelling), equations are estimated for each outcome variable cut-point on an ordered scale but only one parameter estimate is reported for each predictor variable, carrying the assumption that the parameter does not differ substantially between equations. This so-called assumption of parallel regression was checked for reported odds ratios (ORs) (Snedker et al. 2002) using the Brant test, which is available for the OLOGIT command in STATA. Reported parameter estimates were exponentiated to provide equivalent 'averaged' ORs for ease of presentation in a standardized form. Due to the strong relationship between the baseline score on the CIS-R and outcome we adjusted the OR estimates for age and CIS-R score at time 1 and sex (in analyses where the sexes were combined). The recommended CIS-R threshold score for significant psychiatric morbidity is $\geqslant 12$ (Lewis et al. 1992), which was used to divide the sample at time 1 into case and noncase subgroups for tests of interaction with baseline case status and for subgroup analyses. Planned tests of interaction between gender, baseline case status and primary group size were carried out in accord with the stated hypotheses. An examination of possible reverse causality was also carried out by modelling the effect of time 1 symptoms on time 2 small primary group size.

\section{RESULTS}

\section{Survey response rates}

The response rate was $68 \%$. Interviews were not possible for 492 people (14\% of the sample). Interviews were obtained from 2406 people, $79 \%$ of those contacted. There were very few statistically significant differences between responders $(n=2406)$ and non-responders $(n=$ 1900 ) to this follow-up (Singleton \& Lewis, 2003). As occurs in most surveys, young, single people were slightly less likely to respond, while married couples with children were more likely to do so. There was also a slightly higher nonresponse rate in urban areas. Non-responders were also more likely to be renting their 
Table 1. Ordinal logistic regression: effect of small primary group at time 1 on time 2 CIS-R grouped total score in male and female subset analyses in time 1 cases and non-cases

\begin{tabular}{|c|c|c|c|}
\hline $\begin{array}{l}\text { Size of primary group } \\
\text { at time } 1\end{array}$ & Base & Adj. OR & $95 \% \mathrm{CI}$ \\
\hline Men (non-cases) & 742 & & \\
\hline$\geqslant 9$ & & $1 \cdot 00$ & - \\
\hline $4-8$ & & $0 \cdot 97$ & $0 \cdot 50-1 \cdot 64$ \\
\hline $0-3$ & & $4 \cdot 46^{* *}$ & $1 \cdot 90-10 \cdot 47$ \\
\hline Men (cases) & 274 & & \\
\hline$\geqslant 9$ & & $1 \cdot 00$ & - \\
\hline 4-8 & & $1 \cdot 43$ & $0 \cdot 77-2 \cdot 65$ \\
\hline $0-3$ & & $0 \cdot 92$ & $0 \cdot 32-2 \cdot 62$ \\
\hline Women (non-cases) & 907 & & \\
\hline$\geqslant 9$ & & $1 \cdot 00$ & - \\
\hline $4-8$ & & 1.06 & $0.63-1.76$ \\
\hline $0-3$ & & $1 \cdot 49$ & $0 \cdot 65-3 \cdot 41$ \\
\hline Women (cases) & 274 & & \\
\hline$\geqslant 9$ & & $1 \cdot 00$ & - \\
\hline 4-8 & & $1 \cdot 26$ & $0 \cdot 82-1 \cdot 93$ \\
\hline $0-3$ & & $2 \cdot 94 * *$ & $1 \cdot 47-6 \cdot 88$ \\
\hline
\end{tabular}

Adj. OR, adjusted odds ratio; CI, confidence interval.

Dependent variable CIS-R total score at time 2 in four groups: $0-5,6-11,12-17, \geqslant 18$. Analyses adjust for age, sex and CIS-R group level at baseline as appropriate.

** $p<0 \cdot 01$.

accommodation, to lack educational qualifications, and to have lower incomes than responders. A total of $31 \%$ of respondents were cases (CIS-R score of $\geqslant 12$ ) at time 1 and $34 \%$ of non-responders were cases (Singleton \& Lewis, 2003).

\section{Model results}

The effect on future mental health of having a small primary group differed according to gender and according to whether or not the respondent was a case at time 1 (Table 1). These analyses, stratified by gender and case status at time 1, show averaged ORs for level of CMD at time 2 adjusted for time 1 level on the CIS-R, age and sex as appropriate. A primary group size of $0-3$ was significantly associated with time 2 CIS-R level in women who were cases at time 1 (adjusted OR 2.9, 95\% CI 1.5-6.9), but not in male cases (adjusted OR 0.9, 95\% CI 0.3-2.6). The analyses in men and women who were noncases at time 1 (Table 1 ) showed that a primary group size of $0-3$ was strongly associated in men (OR adjusting for CIS-R level and age at baseline $4 \cdot 5,95 \%$ CI $1 \cdot 9-10 \cdot 5$ ), but not in women (adjusted OR 1.5, 95\% CI 0.7-3.4). Thus, in two of the four subgroups, the effect of small primary group size was statistically significant (Table 1).

In these two significant subgroups we re-ran these models after adjusting for severe lack of perceived social support assessed from respondents' answers to seven questions taken from the 1987 Health and Lifestyle survey (Cox et al. 1987), while adjusting for age and severity of CMD at time 1 . In male non-cases at time 1, the adjusted OR for small primary group size increased slightly to $5 \cdot 1$. In female cases at time 1 , after controlling for the same confounders including severe lack of perceived social support, the OR for small primary group size remained statistically significant at the $5 \%$ level but dropped to $2 \cdot 6(95 \%$ CI $1 \cdot 2-5 \cdot 3)$. The crude OR (without adjusting for any potential confounders) was unchanged in male non-cases but in female cases the crude OR for time 1 small primary group size was slightly greater $(3 \cdot 5,95 \%$ CI $1 \cdot 9-6 \cdot 5)$.

The final subgroup analyses considered the possible effect of severe lack of perceived social support after controlling for small primary group size and the other confounders in the same two subgroups: the effect of severe lack of perceived social support at time 1 on CMD at time 2 was not statistically significant.

Outcome was examined in four models of interactions between a small primary group size with gender and with baseline mental status in Table 2. Of the four tests for statistical interaction carried out only one test was clearly not statistically significant, interaction with baseline case status in women (Table 2). These interactions are not completely explored in the subgroup analyses in Table 1 because the effect of gender and of time $1 \mathrm{CMD}$ on CMD at time 2 could also vary according to the size of the primary group. There was no evidence for differences in the effect of time 1 case status in those with a small primary group size compared with larger sizes. However, in non-cases at time 1 the effect of gender on CMD at time 2 was found to vary by size of primary group: female gender was protective when primary group size was small (OR $0 \cdot 24,95 \%$ CI $0.07-0.82$, $p<0.05)$ and increased risk of time 2 CMD when primary group size was large (OR 1.80, $95 \%$ CI $1 \cdot 30-2 \cdot 51, p<0 \cdot 01)$.

Subgroup analyses and tests for interaction were also carried out for severe lack of perceived 
Table 2. Ordinal logistic regression: effect of small primary group at time 1 on time $2 C I S-R$ grouped total score showing interaction tests with gender and with time 1 case status

\begin{tabular}{|c|c|c|c|}
\hline Size of primary group at time 1 & Base & OR & $95 \% \mathrm{CI}$ \\
\hline $\begin{array}{l}\text { Interaction by whether case or } \\
\text { well at time } 1: \text { men only }\end{array}$ & 1016 & & \\
\hline$\geqslant 9$ & & $1 \cdot 00$ & - \\
\hline $4-8$ & & 0.69 & $0 \cdot 26-1 \cdot 85$ \\
\hline $0-3$ & & $5 \cdot 53^{*}$ & $1 \cdot 40-21.98$ \\
\hline $\begin{array}{l}\text { Interaction by whether case or } \\
\text { well at time } 1 \text { : women only }\end{array}$ & 1377 & & \\
\hline$\geqslant 9$ & & $1 \cdot 00$ & - \\
\hline 4-8 & & $0 \cdot 88$ & $0.45-1.69$ \\
\hline $0-3$ & & $0 \cdot 51$ & $0 \cdot 63-1 \cdot 44$ \\
\hline $\begin{array}{l}\text { Interaction by gender: } \\
\text { non-cases at time } 1\end{array}$ & 1649 & & \\
\hline$\geqslant 9$ & & $1 \cdot 00$ & - \\
\hline 4-8 & & $1 \cdot 13$ & $0 \cdot 50-2 \cdot 55$ \\
\hline $0-3$ & & $0 \cdot 31^{*}$ & $0 \cdot 10-0 \cdot 99$ \\
\hline $\begin{array}{l}\text { Interaction by gender: } \\
\text { cases only at time } 1\end{array}$ & 744 & & \\
\hline$\geqslant 9$ & & $1 \cdot 00$ & - \\
\hline 4-8 & & $0 \cdot 91$ & $0 \cdot 41-2 \cdot 06$ \\
\hline $0-3$ & & $3 \cdot 11$ & $0 \cdot 87-11 \cdot 13$ \\
\hline
\end{tabular}

OR, Odds ratio; CI, confidence interval.

All analyses adjust for: gender, age, time 1 CIS-R level as appropriate. Dependent variable CIS-R total score at time 2 in four groups: $0-5,6-11,12-17, \geqslant 18$

$* p<0 \cdot 05$.

social support (primary group size was not included). In one subgroup, male non-cases, the effect of perceived lack of social support was clearly not significant and in the other male subgroup, cases at time 1 , it was borderline $(p=0 \cdot 06)$. In both female subgroups the effect of perceived lack of social support on time 2 CMD was clearly statistically significant. However, tests for interaction between severe lack of social support by both baseline mental status and by gender were clearly not statistically significant.

Our final analysis examined predictors of small primary group size. In men and women baseline mental status at time 1 was significantly associated with small primary group size at time 2 (Table 3). The effect appeared to be greater in women but there was no statistical interaction with gender. Following adjustment for primary group size at time 1 the effect of time 1 CIS-R mental status continued to be strong and statistically significant in women and was of borderline statistical significance in men (Table 3 ).

The assumption of parallel regression test as to be expected was significant for gender and for the CIS-R case threshold when both groups were modelled, but these were addressed by the stratified analyses in which all but one of the tests was negative. All the tests were not significant for small primary group size. The test for severe lack of perceived social support was significant in one subgroup only, female non-cases $(p=0 \cdot 04)$.

\section{DISCUSSION}

\section{Summary of findings}

We report new data supporting earlier findings that the relation of small primary group size to subsequent mental health is conditional on gender and baseline mental health status. In subgroup analyses, the association of small primary group size with future mental health was conditional upon gender and baseline case status revealing strong effects in men who were non-cases and in women who were cases (Table 1) but not in the other two subgroups. More stringent formal tests for interaction with gender and baseline status, although less clear cut, showed statistically significant findings in men for interaction with baseline mental status and in non-cases for interaction with gender at time 1 (Table 2). In general, the relationship of perceived social support at time 1 to symptoms at time 2 did not differ significantly between men and women. The effect of a small primary group size at time 1 on the severity of psychiatric disorder at time 2 in male non-cases and female cases remained statistically significant after controlling for time 1 case status and for other confounders measured at time 1, age and perceived social support. Baseline mental status independently predicted follow-up primary group size in women but not in men when the effect of time 1 primary group size was adjusted for.

\section{Study limitations}

A common limitation in longitudinal research is the difficulty of quantifying the effect of sample attrition. However, in this study there were very few significant differences between responders and non-responders (Singleton \& Lewis, 2003), the latter tending to be younger and more likely to be urban dwellers. The study also had limited 
Table 3. Survey logistic regression: crude effect of CIS-R case status at time 1 on small primary group at time 2, and effect following adjustment for small primary group at time 1 in men and in women

\begin{tabular}{|c|c|c|c|c|c|}
\hline & Predictor & Unadjusted OR & $\begin{array}{c}\text { Regression } \\
95 \% \mathrm{CI}\end{array}$ & Adjusted OR & $\begin{array}{c}\text { Regression } \\
95 \% \mathrm{CI}\end{array}$ \\
\hline \multicolumn{6}{|l|}{$\operatorname{Men}(n=1016)$} \\
\hline \multirow[t]{2}{*}{ CIS- $R$ case status, time 1} & Case & $2 \cdot 50^{* *}$ & $1 \cdot 38-4 \cdot 52$ & 1.88 & $0 \cdot 95-3 \cdot 71$ \\
\hline & Non-case & $1 \cdot 00$ & - & $1 \cdot 00$ & - \\
\hline \multirow{2}{*}{ Small primary group, time 1} & Present & - & - & $17 \cdot 96^{* * *}$ & $8 \cdot 10-39 \cdot 86$ \\
\hline & Absent & - & - & $1 \cdot 00$ & - \\
\hline \multicolumn{6}{|l|}{ Women $(n=1375)$} \\
\hline \multirow[t]{2}{*}{ CIS- $R$ case status, time 1} & Case & $4.92 * * *$ & $2 \cdot 74-8 \cdot 83$ & $3 \cdot 61^{* * *}$ & $1 \cdot 88-6.94$ \\
\hline & Non-case & $1 \cdot 00$ & - & $1 \cdot 00$ & - \\
\hline \multirow[t]{2}{*}{ Small primary group, time 1} & Present & - & - & $20 \cdot 42 * * *$ & $9 \cdot 10-46 \cdot 04$ \\
\hline & Absent & - & - & $1 \cdot 00$ & - \\
\hline
\end{tabular}

OR, Odds ratio; $\mathrm{CI}$, confidence interval.

** $p<0 \cdot 01, * * * \quad p<0 \cdot 001$.

power to detect potentially useful effects in smaller subgroups such as cases. This applies particularly to tests for statistical interaction. This is one of the reasons for reporting subgroup analyses as well as interactions, so that the weight of non-significant interaction tests can be judged.

More generally, there are limits to what can be achieved by epidemiological studies because it is not feasible to conduct very detailed assessments of social and mental health functioning. For example, our finding that size of social network predicts future mental health after controlling for a measure of perceived social support may suggest that both measures assess social support in distinctly different and not clearly understood ways. Other approaches to studying gender and social relationships would be of value, employing qualitative methods and behavioural observation methods.

Another possible limitation in the present study was that measures of risk factors and neurotic symptoms were based only on precisely prescribed questions with no allowance for follow-up enquiry (Brugha et al. 1999). The mental health assessment used in the present analyses may be open to further criticism. A particular limitation of this study is the lack of detailed information on the timing of symptom recovery and development both during the 18-month interval between assessments and in the past. One possible objection is that individuals who were symptom free at both assessments might have become symptomatic and have recovered between the assessments and, thus, would have been incorrectly classified in our models. However, accurate information on the origins, development and remission of symptoms would have to rely either on retrospective data collection, with its attendant greater unreliability (Andrews et al. 1999), or on frequent re-assessments from an early age that impose a considerable burden of cooperation.

Many critical issues have been raised by research findings on social support and mental disorder. Early development (Champion, 1995) and personality traits (Alloway \& Bebbington, 1987) may be important determinants of social support for which we did not use standard measures in this study. However, it was possible to consider whether effects operate in both directions. The difference between the unadjusted and adjusted models of follow-up symptom severity attributable to small primary group size suggest that the effect of primary group size at time 1 on future mental disorder is partly attributable to symptoms assessed at that time, at least in women. However, even when time 1 symptoms are taken into account small primary group size is significant in predicting future symptom levels. The finding that future mental health status is predicted, even when a range of potential confounders is adjusted for, deals with the argument that a small primary group size follows from and does not influence mental disorder. While the effect of baseline primary group size on later primary group size is both considerable and as one would expect highly significant, that of prior symptoms appears be small in comparison and it differs somewhat 
between men and women. In men only, when baseline primary group size is adjusted for, the effect of baseline mental status on small primary group size at outcome is of borderline significance. Nevertheless, longer-term follow-up studies are needed to examine the primary effect of symptom expression on the size of personal social networks and vice versa from the earliest development of each, which would be particularly valuable if begun before the beginning of male and female adolescence.

\section{Interpretation of findings}

We found that small primary group size is a predictor of mental health even when severe lack of perceived social support is adjusted for, which suggests that these two predictors are relatively independent of one another in their effects on later CMD. Most measures of social support tend to capture satisfaction with, and the adequacy of personal relations, and ask the respondent to conduct an appraisal of supportiveness from others (Lakey \& Cohen, 2000). Even where social relationships are studied, the focus tends to be on relationship qualities such as companionship, conflict, intimacy, rather than on the number of relationships. The present and previous studies of primary group size have their origins in social integration (Brissette et al. 2000) and social network theory. In this, network size and other structural properties of networks are of interest (Mitchell, 1969). This structural approach is infrequently used in studies of physical or mental health. Mean network size has been studied in various animal species, suggesting species-specific as well as context- and environment-specific influences (Wilson, 1975). Network size may be a valuable paradigm for integrating social and biomedical research (Brissette et al. 2000).

Our findings are complex but confirm similar patterns of association in different study samples over a 15 -year period. The pattern of conditional associations seen in subgroup analyses, partially supported by the more stringent statistical tests of interaction, pose intriguing questions of interpretation that have implications for research on social functioning in psychiatry. Some have been discussed before. With regard to gender, women do not differ substantially from men in the size of their primary groups (Brugha et al. 1990; Meltzer et al. 1995b). Brugha and colleagues (1990), however, speculated only when women develop a significant spell of disorder do they begin to discover who really can be relied upon, that is close as distinct from 'fair-weather' friends. Mentally unwell women describing their close network may, therefore, be revealing a less optimistic but more accurate account; when they describe a small primary group size this may be more likely to accurately reflect a more limited range of support sources. Brugha and colleagues (1998) found that primary group size fails significantly to predict future mental ill health in women in the community, which would appear to fit with this perspective.

However, in men the findings were clearly and precisely opposite. This tallies with literature quoted in the Introduction to this paper and with the finding of Brugha et al. (1990) that the outcome of depression in men was not predicted by the number of close others, although it was predicted by the number of non-close acquaintances and casual social contacts in the previous week (Brugha et al. 1990). Why should primary group size assessed when relatively symptom free so clearly explain future mental health in men but not in women? The suggestion that mentally well men are more discerning than women about whom they can count on as close seems counter-intuitive. However, the possibility that they are less discerning when mentally unwell may be more plausible. Women are more often tied by family and social obligation, and even when emotionally unwell or 'let down' by others are less free to walk away, particularly when they have care responsibilities for children or disabled relatives. The post-hoc finding that increased risk of poor mental health at time 2 in women appears to apply to non-cases at time 1 with a larger primary group size (which needs to be replicated) also draws attention to the possible potential for close relationships to be burdensome. Not being so easily able to evade responsibilities may mean that women with CMD learn more quickly who among their close others can be relied upon, because they have little choice. Men may display more irritability and anti-social behaviour when mentally unwell and, therefore, may not rely on close others - perhaps, seeking and drawing upon less formal and other sources of support. The size of 
a man's primary group may also be a function of prestige and position in the social hierarchy that is protective in the face of adversity. Many other explanations could be put forward, for example based on social role theory (Eagley, 1987) - the key issue is that research is needed to explore the underlying explanations for these consistent findings.

In conclusion, in those who are not cases of $\mathrm{CMD}$ in the general population we have demonstrated a striking difference between the sexes in the effect of having a small primary group on future mental health that is not explained by adjustment for other potential confounders or indeed by perceived social support. In spite of a considerable literature on social support for over two decades very little attention has been given to the potentially more objective role of social network structure and the possible role of such statistical interactions. Hopefully these findings will now stimulate renewed interest and enquiry into the origins of CMD.

\section{DECLARATION OF INTEREST}

None.

\section{REFERENCES}

Alloway, R. \& Bebbington, P. (1987). The buffer theory of social support - a review of the literature. Psychological Medicine 17, $1-108$.

Andrews, G., Anstey, K., Brodaty, H., Issakidis, C. \& Luscombe, G. (1999). Recall of depressive episode 25 years previously. Psychological Medicine 29, 787-791.

Brissette, I., Cohen, S. \& Seeman, T. E. (2000). Measuring social integration and social networks. In Social Support Measurement and Intervention: a Guide for Health and Social Scientists (ed. S. Cohen, L. G. Underwood and B. H. Gottlieb), pp. 53-85. Oxford University Press: New York.

Brugha, T. S., Bebbington, P. E. \& Jenkins, R. (1999). A difference that matters: comparisons of structured and semi-structured diagnostic interviews of adults in the general population. Psychological Medicine 29, 1013-1020.

Brugha, T. S., Bebbington, P. E., MacCarthy, B., Sturt, E., Wykes, T. \& Potter, J. (1990). Gender, social support and recovery from depressive disorders: a prospective clinical study. Psychological Medicine 20, 147-156.

Brugha, T. \& Britto, D. (1992). Social support: heredity, environment and conditional associations. Current Opinion in Psychiatry $\mathbf{5}$, 305-308.

Brugha, T., Conroy, R., Walsh, N., Delaney, W., O'Hanlon, J., Dondero, E., Daly, L., Hickey, N. \& Bourke, G. (1982). Social networks, attachments and support in minor affective disorders: a replication. British Journal of Psychiatry 141, 249-255.

Brugha, T. S., Morgan, Z., Bebbington, P., Jenkins, R., Lewis, G., Farrell, M. \& Meltzer, H. (2003). Social support networks and type of neurotic symptom among adults in British households. Psychological Medicine 33, 307-318.
Brugha, T. S., Sharp, H. M., Cooper, S. A., Weisender, C., Britto, D., Shinkwin, R., Sherrif, T. \& Kirwan, P. H. (1998). The Leicester 500 Project. Social support and the development of postnatal depressive symptoms, a prospective cohort survey. Psychological Medicine 28, 63-79.

Brugha, T. S., Sturt, E., MacCarthy, B., Potter, J., Wykes, T. \& Bebbington, P. E. (1987). The Interview Measure of Social Relationships: the description and evaluation of a survey instrument for assessing personal social resources. Social Psychiatry 22, 123-128.

Brugha, T. S., Wing, J. K., Brewin, C. R., MacCarthy, B. \& Lesage, A. (1993). The relationship of social network deficits with deficits in social functioning in long-term psychiatric disorders. Social Psychiatry and Psychiatric Epidemiology 28, 218-224.

Champion, L. (1995). A developmental perspective on social support networks. In Social Support and Psychiatric Disorder: Research Findings and Guidelines for Clinical Practice (ed. T. S. Brugha), pp. 61-95. Cambridge University Press: Cambridge.

Cox, B. D., Blaxter, M., Buckle, A. L. J., Fenner, N. P., Golding, J. F., Gore, M., Huppert, F. A., Nickson, J., Roth, M., Stark, J., Wadsworth, M. E. J. \& Wichelow, M. (1987). The Health and Lifestyle Survey. Health Promotion Research Trust.

Eagley, A. H. (1987). Sex Differences in Social Behaviour. Erlbaum: Hilsdale, NJ

Henderson, S., Duncan Jones, P, McAuley, H. \& Ritchie, K. (1978). The patient's primary group. British Journal of Psychiatry $\mathbf{1 3 2}$, $1-86$.

Hintikka, J., Koskela, T., Kontula, O., Koskela, K. \& Viinamaki, H. (2000). Men, women and friends - are there differences in relation to mental well-being? Quality of Life Research 9, 841-845.

Kawachi, I. \& Berkman, L. F. (2001). Social ties and mental health. Journal of Urban Health: Bulletin of the New York Academy of Medicine 78, 458-467.

Lakey, B. \& Cohen, S. (2000). Social support theory and measurement. In Social Support Measurement and Intervention: a Guide for Health and Social Scientists (ed. S. Cohen, L. G. Underwood and B. H. Gottlieb), pp. 29-52. Oxford University Press: New York.

Lewis, G., Pelosi, A. J., Araya, R. \& Dunn, G. (1992). Measuring psychiatric disorder in the community: a standardized assessment for use by lay interviewers. Psychological Medicine 22, 465-486.

Meltzer, H., Gill, B., Petticrew, M. \& Hinds, K. (eds.) (1995a). Office of Population Censuses \& Surveys Social Survey Division. OPCS Surveys of Psychiatric Morbidity in Great Britain. Report 1: The prevalence of psychiatric morbidity among adults living in private households, 101 pp. OPCS Surveys of Psychiatric Morbidity in Great Britain. Office for National Statistics Social Survey Division.

Meltzer, H., Gill, B., Petticrew, M. \& Hinds, K. (eds.) (1995b). Office of Population Censuses \& Surveys Social Survey Division. OPCS Surveys of Psychiatric Morbidity in Great Britain. Report 3: Economic activity and social functioning of adults with psychiatric disorders. HMSO, 168 pp. OPCS Surveys of Psychiatric Morbidity in Great Britain. HMSO.

Meltzer, H., Singleton, N, Lee, A., Bebbington, P., Brugha, T. \& Jenkins, R. (eds.) (2002). Office for National Statistics Socia Survey Division. The Social and Economic Circumstances of Adults with Mental Disorders. The Stationery Office, 87 pp.

Mitchell, J. C. (1969). The concept and use of social networks. In Social Networks in Urban Situations (ed. J.C. Mitchell), Manchester University Press: Manchester.

ONS (2004). National Statistics Online (www.statistics.gov.uk). Office for National Statistics.

Paykel, E. S. \& Weissman, M. M. (1973). Social adjustment and depression. A longitudinal study. Archives of General Psychiatry 28, 659-663.

Singleton, N., Bumpstead, R., O'Brien, M., Lee, A. \& Meltzer, H. (eds.) (2001). National Statistics. Psychiatric Morbidity Among Adults Living in Private Households. The Stationery Office, $154 \mathrm{pp}$. 
Singleton, N. \& Lewis, G. (eds.) (2003). Better or worse: a longitudinal study of the mental health of adults living in private households in Great Britain. The Stationery Office.

Snedker, K., Glynn, P. \& Wang, C. (2002). Ordered/ordinal logistic regression with SAS and Stata (http://www.staff.washington.edu/ glynn/olr.pdf). Document prepared by the authors. University of Washington, DC, USA. Acessed 12 June 2003.

StataCorp (1999). STATA statistical software: release 6.0. Stata Corporation: College Station, TX.
Stokes, J. \& Levin, I. (1986). Gender Differences in predicting loneliness from social network characteristics. Journal of Personality and Social Psychology 51, 1069-1074.

Wildes, J. E., Harkness, K. L. \& Simons, A. D. (2002). Life events, number of social relationships, and twelve-month naturalistic course of major depression in a community sample of women. Depression and Anxiety 16, 104-113.

Wilson, E. O. (1975). Sociobiology: The New Synthesis. Belknap Press of Harvard University Pres. : Cambridge, MA. 